\title{
MEDICAMENTOS NA PRÁTICA CLÍNICA DE PACIENTES ONCOLÓGICOS EM CUIDADOS PALIATIVOS ${ }^{1}$
}

\author{
MEDICINES IN THE CLINICAL PRACTICE OF \\ ONCOLOGICAL PATIENTS IN PALLIATIVE CARE
}

\author{
Lusiéte Maurer Ferrari² $^{2}$ Jane Beatriz Limberger ${ }^{3}$
}

\section{RESUMO}

Introdução: Os cuidados paliativos melhoram a qualidade de vida dos pacientes e de seus familiares que enfrentam problemas associados a doenças potencialmente fatais, sejam elas físicas, psicológicas, sociais ou espirituais. Quando direcionado aos pacientes oncológicos é um tratamento essencial, pois propicia conforto e ainda visa priorizar a dignidade e valorizar os doentes de forma humanizada e holística. Objetivo: Buscou-se neste trabalho discutir e identificar as principais medicações descritas na literatura e utilizadas na prática clínica de pacientes oncológicos em cuidados paliativos, assim como suas indicações. Metodologia: Foi realizada uma pesquisa bibliográfica, na qual utilizou-se dissertações, manuais, Diretrizes do Ministério da Saúde e artigos científicos pesquisados em bases de dados como Pubmed, Science Direct e Google Scholar por meio dos descritores: cuidados de conforto, práticas complementares, cancerologia, dor oncológica, oncologia integrativa. Resultados: Neste artigo foram abordados os cuidados paliativos nos tratamentos dos sinais e sintomas de problemas respiratórios, náuseas e vômitos, constipação intestinal e diarreia, delirium, distúrbios hemorrágicos e a dor de pacientes oncológicos. Os resultados apontam a necessidade da correta identificação dos sintomas para a escolha adequada do tratamento farmacológico para estas condições, e a importância da equipe multidisciplinar para o seguimento farmacoterapêutico no manejo de complicações da doença e dos tratamentos. Conclusão: Conclui-se, então, a importância dos cuidados paliativos para os sinais e sintomas do câncer avançado, da necessidade de analisar e avaliar os sintomas de cada paciente a fim de realizar uma melhor prescrição e administração de cada medicamento.

Palavras-chave: Cancerologia, Cuidados de conforto, Dor oncológica, Práticas complementares, Oncologia integrativa.

\section{ABSTRACT}

Introduction: Palliative care is defined as active and comprehensive health care provided to people with severe and progressive and / or terminal illness. This assistance aims to promote the quality of life of patients and their families, and when targeted at cancer patients, it is an essential treatment, as it leads to comfort, and aims to prioritize dignity and value patients in a humanized and holistic way. Objective: This study sought to discuss and identify the main medications described in the literature and used in the clinical practice of cancer patients in palliative care, as well as their indications. Methodology: A bibliographic search was carried out, using dissertations and scientific articles researched in databases such as Pubmed, Science Direct and Google Scholar through the descriptors: comfort care, complementary practices, cancerology, cancer pain, integrative oncology. Results: In this article, palliative care was addressed in the treatment of signs and symptoms of respiratory problems, nausea, constipation, diarrhea, delirium, circulatory disorders, and pain.

1 Trabalho Final de Graduação

2 Estudante do curso de Farmácia, Universidade Franciscana. E-mail: lusiete.ferrari@ufn.edu.br

3 Orientadora. Docente do curso de Farmácia, Universidade Franciscana. E-mail: janebeatriz@ufn.edu.br 
The results point to the need for correct identification of symptoms for the proper choice of pharmacological treatment for these conditions, and the importance of pharmacotherapeutic follow-up in the management of disease complications and treatments. Conclusion: It is concluded, then, the importance of palliative care for the signs and symptoms of advanced cancer, the need to analyze and evaluate the symptoms of each patient to perform a better prescription and administration of each medication.

Keywords: Cancerology, Comfort care, Complementary practices, Integrative oncology, Oncological pain.

\section{INTRODUÇÃO}

Segundo a definição da Organização Mundial de Saúde - OMS, publicada em 1990 e revisada em 2002 e 2017, Cuidado Paliativo é uma abordagem que melhora a qualidade de vida de pacientes (adultos e crianças) e seus familiares, que enfrentam desafios associados a doenças que ameaçam a vida. Essa assistência previne e alivia o sofrimento, através da identificação precoce, avaliação correta e tratamento da dor e de outros problemas físicos, psicossociais ou espirituais (KOHLER; CERCHIARO; LEVITES, 2016; WHO, 2020).

A equipe de cuidados paliativos acolhe pacientes com diversas enfermidades que ameacem a continuidade da vida, desde casos simples, como por exemplo a hipertensão, onde o paciente deverá mudar alguns hábitos de vida, até casos mais graves, como problemas pulmonares, insuficiência cardíaca, doenças crônicas e câncer, onde o cuidado paliativo se faz ainda mais necessário. $\mathrm{O}$ foco dessa assistência consiste em assegurar uma melhor qualidade de vida e bem-estar do paciente e de seus familiares, buscando alternativas para minimizar os sintomas de desconforto, sejam eles causados pela doença ou pelo tratamento, até o momento da morte, preparando os entes para o processo do luto (LANZA et al., 2015; NOGUEIRA, 2019; SOUZA et al., 2016).

Para conseguir melhorar a qualidade de vida do paciente em cuidado paliativo, muitos medicamentos como analgésicos, psicotrópicos, antiepiléticos e antieméticos são utilizados no controle sintomático. No entanto, a farmacocinética e farmacodinâmica destes fármacos pode estar alterada nos pacientes paliativos em decorrência de comorbidades e alterações fisiológicas que ocorrem, principalmente, quando a pessoa se encontra em fase terminal. As mudanças na farmacocinética e farmacodinâmica podem causar aumento ou diminuição das concentrações sanguíneas do medicamento, o que pode resultar em eficácia alterada ou aumento do risco de reações adversas ao medicamento. E quanto à farmacogenômica, a variabilidade genética pode afetar a resposta de um indivíduo ao tratamento com drogas por influenciar os processos farmacocinéticos e farmacodinâmicos (FRANKEN et al., 2016; KREMER, 2015; NOGUEIRA, 2019; ROBERTS et al., 2018).

Em função dessas alterações, é crucial a reavaliação da terapêutica e elaboração de um plano farmacoterapêutico adaptado às necessidades individuais de cada paciente, que deve ser feito por uma equipe multidisciplinar, na qual o parecer de um farmacêutico é bastante significativo. A assistência farmacêutica em cuidados paliativos desempenha o papel de fornecer medicamentos que promovam 
o controle dos sintomas do paciente, com a finalidade de obter resultados positivos no tratamento e proporcionar qualidade de vida por meio da melhoria do curso da doença. Além disso, participa do processo de tomada de decisão sobre a farmacoterapia e acompanhar o paciente durante a terapia farmacológica de maneira preventiva, a fim de evitar e minimizar os problemas relacionados aos medicamentos, visando assim um tratamento mais seguro (LOBATO et al., 2019; SOUZA et al., 2016).

O presente trabalho teve como objetivo realizar revisão de literatura acerca de publicações relacionadas aos principais medicamentos utilizados na prática clínica de pacientes oncológicos em cuidado paliativo para controle de sintomas, assim como seus efeitos adversos. A fim de colaborar para melhora da assistência prestada aos pacientes em cuidados paliativos, e para a qualificação acadêmica e profissional frente ao assunto medicações em cuidados paliativos.

\section{MATERIAL E MÉTODOS}

Foi realizada uma pesquisa bibliográfica a partir de artigos, livros e publicações em base de dados Pubmed, Science direct, Google scholar, além de Cadernos, Manuais e Diretrizes do Ministério da Saúde. Foram utilizados descritores como: cuidados de conforto, práticas complementares, cancerologia, dor oncológica, oncologia integrativa. A partir dos artigos encontrados dos últimos cinco anos, foram levantadas as seguintes temáticas que serviram de base para a discussão: Manejo dos problemas respiratórios; Manejo da náusea e vômito; Manejo da constipação e diarreia; Manejo do delirium; Manejo dos distúrbios hemorrágicos e Manejo da dor.

\section{RESULTADOS E DISCUSSÃo}

\section{MANEJO DOS SINTOMAS COMUNS EM PACIENTES ONCOLÓGICOS PALIATIVOS}

Pacientes com câncer em sua fase inicial passam por tratamentos agressivos, com o objetivo de alcançar a cura. Porém, quando a doença está em estágio avançado e o tratamento para a cura não é eficaz, a abordagem paliativa deve ser utilizada para manejo dos sintomas de difícil controle e alguns aspectos psicossociais relacionados à doença. O cuidado paliativo no paciente oncológico demanda atenção integral, incluindo prevenção, detecção precoce, diagnóstico e tratamento adequado, com a finalidade de promover resultados satisfatórios para o paciente, melhorando a sua qualidade de vida (ZOCCOLI et al., 2019).

A terapêutica dos sintomas decorrentes da doença ou do tratamento do paciente em cuidado paliativo exige diversos tratamentos combinados ou medidas não farmacológicas, o que é uma estratégia fundamental em todos os estágios. O plano terapêutico deve ser individualizado e focado nas necessidades de cada paciente. Desta forma, para o controle destes sintomas se faz necessário a 
existência de uma equipe multidisciplinar no acompanhamento do paciente durante todo o tratamento. Por mais que existam especificidades de cada área profissional, todos que atuam devem ser capazes de reconhecer sintomas e compreender maneiras básicas de manejo e/ou seus encaminhamentos (BAZANTE, 2016; GOMES; OTHERO, 2016; GUERRA et al., 2019; ZOCCOLI et al., 2019).

Esta equipe multidisciplinar é constituída por médicos, enfermeiros, nutricionistas, psicólogos, psiquiatras, terapeutas ocupacionais, entre outros profissionais. Recentemente, o farmacêutico conquistou seu espaço, tornando-se primordial na qualidade do processo farmacoterapêutico. A inclusão de uma equipe de farmacêuticos na equipe de cuidados paliativos é benéfica, pois os cuidados farmacêuticos constituem-se na prestação de serviços que envolvem a dispensação e utilização de medicamentos, com a finalidade de contribuir e otimizar os planos de cuidado do paciente, obtendo resultados no tratamento que melhorem a qualidade de vida dele e de seus familiares. Na oncologia o farmacêutico participa desde a seleção e padronização, aquisição e conservação dos medicamentos e insumos farmacêuticos, verificando se todos os fatores estão de acordo às exigências legais. Ele também tem a responsabilidade de realizar a análise das prescrições, verificando se a opção do medicamento está correta e se é a de melhor escolha, se faz parte dos protocolos clínicos daquele estabelecimento de saúde, bem como se as doses, embalagens e diluentes mais adequados, além de realizar a manipulação dos fármacos (TEIXEIRA, 2015).

Conforme Lobato e colaboradores (2019), o farmacêutico deve, ainda, promover a adesão do paciente ao tratamento farmacológico, realizar o monitoramento da progressão ou regressão da doença por meio de exames clínicos e físicos, além de realizar intervenções para a promoção da sua saúde. Além disso, devem acompanhar a terapia farmacológica a fim de prevenir e/ou minimizar os problemas relacionados a medicamentos, efeitos adversos, interações medicamentosas e consequentemente, o tempo de internação e os custos da terapêutica (LOBATO et al., 2019).

É necessário que se institua o manejo adequado dos sintomas mais comuns que podem estar presentes no paciente oncológico. Estes sintomas são advindos da doença ou de seus tratamentos, e devem ser tratados de maneira complementar ao plano terapêutico (CALADO; TAVARES; BEZERRA, 2019). Serão abordados a seguir os sinais e os sintomas mais frequentes no paciente oncológico, com enfoque no manejo farmacoterapêutico. Destacam-se os problemas respiratórios, náuseas e vômitos, constipação intestinal e diarreia, delirium, distúrbios hemorrágicos e a dor, a mais frequente e temida no paciente oncológico.

\section{Manejo dos problemas respiratórios}

O manejo dos problemas respiratórios como dispneia e acúmulo de secreções de pacientes em cuidados paliativos é de grande importância. A prevalência da dispneia em pacientes oncológicos situa-se em torno de 90\%, agravando com a evolução da doença, sendo ainda mais presente em 
pacientes com câncer de pulmão. A dispneia é classificada como uma experiência subjetiva de desconforto respiratório, consistindo em sensações qualitativamente distintas com intensidades diferentes. A experiência origina-se a partir das interações entre uma variedade de fatores físicos (caquexia, astenia muscular); psicológicos (depressão/ansiedade); sociais e ambientais, que podem induzir respostas comportamentais e fisiológicas secundárias. Além disso, é uma das principais causas de perda de função em pacientes com câncer, quando em estágio avançado leva ainda mais impacto na qualidade de vida do paciente e dos seus familiares (ALVES, 2016; ALVES; PINA, 2018; CRUZ; OLIVEIRA; CAPELAS, 2017; OLIVEIRA; MEDEIROS, 2020).

Por ser uma experiência subjetiva, apenas a pessoa com este sintoma está em posição de determinar a sua presença e intensidade da gravidade. Com isto, a autoavaliação da intensidade da dispneia deve ser o método de avaliação utilizado, buscando promover uma terapêutica paliativa adequada. A avaliação clínica tem como objetivo identificar causas subjacentes e avaliar o impacto do desconforto na qualidade de vida do paciente. Existe a indicação para a adoção de métodos complementares para a investigação, tais como a avaliação de aspectos, como por exemplo, a história clínica, exame físico, exames complementares, e ainda causas subjacentes. Para a autoavaliação ainda existem escalas próprias a fim de verificar a intensidade da dispneia, como a escala de avaliação numérica (EAN), que é caracterizada pela objetividade, facilidade de aplicação e compreensão dos resultados. Esta escala apresenta uma régua dividida em onze partes iguais numeradas sucessivamente de 0 a 10 . Ao utilizar-se esta escala, pretende-se que o paciente faça a equivalência entre a intensidade da dispneia e a classificação numérica, onde 0 corresponde sem dispneia e a 10 classifica-se como dispneia insuportável (ALVES; PINA, 2018; RAMOS; TAVARES; MENDONÇA, 2017; ZOCCOLI et al., 2019).

O manejo inicial deste sintoma está no controle da causa de base. Os opioides são considerados de primeira linha para o tratamento farmacológico, e contemplam o uso de morfina e codeína. Também na terapêutica podem ser utilizados benzodiazepínicos como o midazolam, lorazepam e o diazepam, prescritos como medicamentos adjuvantes para aqueles pacientes com crises de dispneia associados a crises de ansiedade ou de pânico. Pode-se, ainda, associar o uso de oxigênio, ventilação não invasiva, e ainda a sedação paliativa no caso de dispneia refratária. É importante atentar para as interações medicamentosas e efeitos adversos desses fármacos, uma vez que o uso prolongado de opioides está relacionado à tolerância e dependência física, e os efeitos adversos mais significativos da morfina são prurido transitório, sonolência, e o mais frequente, constipação, sendo muitas das vezes necessário o uso de laxantes (BAZANTE, 2016; OLIVEIRA; MEDEIROS, 2020; ZOCCOLI et al., 2019).

As intervenções não farmacológicas têm como objetivo melhorar a experiência dos sintomas e abordar as necessidades multidimensionais dos pacientes. Essas medidas consistem em treinamento respiratório, elevação da cabeceira, uso de cadeira de rodas, estimulação neuromuscular elétrica, arejar o ambiente, apoio psíquico, espiritual e social, e ainda algumas técnicas como acupuntura e técnicas de relaxamento (BAZANTE, 2016). 
A hipersecreção das vias aéreas consiste no acúmulo de secreção no trato respiratório e pode ocorrer por diferentes fatores. O excesso dessa secreção nas vias aéreas pode desencadear tosse, dispneia ou ruído respiratório, como visto na fase final de vida. Deste modo, a identificação e o tratamento do sintoma são fundamentais. Existem duas apresentações comuns de hipersecreção de vias aéreas específicos em cuidados paliativos, a sororoca e a broncorreia. A sororoca consiste no ruído que ocorre quando a secreção acumulada em vias aéreas borbulha com a passagem de ar da respiração, comumente nessa fase, o paciente já encontra-se mais debilitado, inconsciente ou semiconsciente. Existem estágios de zero a três onde a intensidade da sororoca pode ser observada no estágio zero não é audível, já no estágio três é claramente audível. Alguns fatores de risco como hidratação artificial excessiva, doenças que prejudicam o reflexo de tosse e deglutição, doenças pulmonares, baixo nível de consciência e infecções podem ajudar na ocorrência da sororoca (ANCP, 2012; ZOCCOLI et al., 2019).

O tratamento farmacológico padrão para sororoca é feito com o uso de antimuscarínicos como escopolamina, que tem como objetivo diminuir a quantidade de secreção produzida. O tratamento precoce é fundamental, porém a terapêutica medicamentosa não consegue eliminar a secreção já acumulada. Já o tratamento não farmacológico inclui medidas como reposicionamento do paciente no leito a cada 3-4h, assim como a elevação de cabeceira, aspiração oral, desde que se apresente efetiva e tolerada. Deve-se evitar aspiração faríngea, pois é desconfortável ao paciente. O uso de música de fundo pode deixar o ambiente mais leve, assim como, pode camuflar o ruído respiratório (ZOCCOLI et al., 2019).

A broncorreia é definida como produção de grande quantidade secreção pulmonar por dia, e o seu tratamento visa o conforto ao paciente. A abordagem farmacológica depende da causa subjacente, e inclui radioterapia paliativa, inalação com brometo de ipratrópio, uso de anticolinérgicos, colírio de atropina, corticosteroides e macrolídeos como eritromicina em casos extremos (OLIVEIRA; MEDEIROS, 2020; ZOCCOLI et al., 2019).

\section{Manejo da náusea e vômito}

A náusea é definida como a sensação de desconforto na região epigástrica seguida de vômitos e expulsão forçada do conteúdo estomacal. Os sintomas gastrointestinais como esses são frequentes em pacientes em cuidados paliativos e estima-se que cerca de $60 \%$ dos pacientes com quadros avançados em oncologia apresentam estes sintomas, que são, na maioria das vezes, induzidos pela quimioterapia. Eles são caracterizados por uma sensação subjetiva desagradável que sinaliza possibilidade de vômito iminente, o qual pode ou não ocorrer, o que acaba causando desconforto e estresse para o paciente. O controle inadequado destes sintomas pode levar a outras complicações, como anorexia, desequilíbrio eletrolítico, desidratação, e ainda a necessidade de hospitalização ou internação prolongada, provocando a diminuição da qualidade de vida e efeitos negativos nas atividades diárias do 
paciente. O tratamento eficaz pode evitar o abandono precoce da adesão ao tratamento, reduzindo assim a morbidade e o risco de complicações (ANDRADE, 2018; BAZANTE, 2016).

Nesse sentido, a êmese causada pela quimioterapia pode ser classificada conforme o momento do seu aparecimento em relação ao tratamento, ela é habitualmente dividida em aguda, tardia, antecipatória, irruptiva e refratária. Para a escolha adequada do antiemético, deve-se considerar o potencial emetogênico (alto, moderado, baixo e mínimo) dos quimioterápicos utilizados e os fatores de riscos específicos do paciente, podendo ser utilizadas associações. Existem, ainda, formas de avaliar o vômito apresentado pelo paciente, como a medição do volume expelido, o número de vezes que o paciente apresenta este sintoma, o tempo entre a administração da quimioterapia e o primeiro vômito, bem como, o tempo que esse efeito pode durar (RAGGHIANTI, 2017).

Os fármacos mais empregados para o controle deste sintoma são antieméticos como os antagonistas da serotonina (ondansetrona, granisetrona, dolasetrona); corticosteroides (dexametasona, metilprednisona); antagonista de neurocinina (aprepitanto); antagonistas da dopamina (metoclopramida, domperidona); e ainda benzodiazepínicos (lorazepam, diazepam). A prevenção adequada é essencial para preservar a qualidade de vida dos pacientes oncológicos. O controle inadequado das náuseas e vômitos agudos poderá implicar no surgimento de náuseas e vômitos tardios, bem como a diminuição da qualidade de vida do paciente e também da sua família (ALMEIDA et al., 2015).

\section{Manejo da constipação e diarreia}

A constipação intestinal pode ser definida como a presença de evacuações dificultosas ou dolorosas associadas a evacuações infrequentes e fezes endurecidas e/ou em pequena quantidade, frequentemente associada à distensão abdominal. A prevalência da constipação em pacientes com câncer avançado varia de $40 \%$ a 90\%, sendo mais comum em pacientes que fazem o uso opioides para controle de sintomas. A prevalência pode aumentar devido à combinação de vários fatores, tais como: doença avançada, idade, redução ou falta de atividade física, fatores dietéticos, e ainda uso de medicamentos obstipantes (CORADAZZI; TARDELLI; CAPONERO, 2019).

A constipação é uma experiência subjetiva, e o critério diagnóstico nem sempre expressa a diversidade de fatores que levam à constipação a ser considerado um sintoma que necessita de manejo adequado. Aspectos como mensurar sintomas objetivamente (característica das fezes, frequência da evacuação), e a percepção do paciente quanto ao desconforto e facilidade ao evacuar devem ser consi-

derados na avaliação clínica. É necessário, ainda, avaliar a percepção individual de cada paciente por meio de anamnese, que pode ser conduzida por meio da avaliação pela Escala de Bristol. Essa escala ajuda o paciente a estimar mais precisamente a forma e características das fezes, facilitando na hora da escolha do manejo terapêutico (LEWIS; HEATON, 1997; ZOCCOLI et al., 2019). 
A ação preventiva e precoce é fundamental e pode incluir tratamento farmacológico ou não. As intervenções não farmacológicas incluem medidas relacionadas à educação e controle dos hábitos alimentares, como, por exemplo, consumo e suplementação de fibras, ingestão adequada de líquidos, uso de alimentos funcionais probióticos; orientação sobre atividades físicas; promoção de conforto e privacidade do paciente durante a evacuação. Já a intervenção farmacológica é realizada com o uso de laxativos, é importante salientar que se deve verificar o possível efeito constipante no início do uso de opioides, para fazer a profilaxia. Os laxantes são classificados conforme seu mecanismo de ação: formadores de bolo (promovem o efeito pela retenção de água na luz intestinal, aumentando o volume das fezes e, assim, estimulando o peristaltismo), osmóticos (por via oral retêm fluidos no lúmen intestinal estimulando o peristaltismo), estimulantes (aumentam o peristaltismo, pelo aumento da secreção de água e eletrólitos pela mucosa intestinal) e emolientes/lubrificantes (aumentam a penetração de água e amolecem as fezes) (CORADAZZI; TARDELLI; CAPONERO, 2019). Os medicamentos mais utilizados estão representados no Quadro 1.

Quadro 1 - Medicamentos mais utilizados no manejo da constipação.

\begin{tabular}{|l|l|}
\hline \multicolumn{1}{|c|}{ TIPO } & \multicolumn{1}{|c|}{ MEDICAMENTO } \\
\hline \multirow{2}{*}{ Formadores de bolo } & Metilcelulose \\
Osílio \\
Osmóticos & Farelo de trigo \\
\hline \multirow{2}{*}{ Estimulantes } & $\begin{array}{l}\text { Hidróxido de Magnésio } \\
\text { Lactulose } \\
\text { Polietilenoglicol }\end{array}$ \\
\hline \multirow{2}{*}{ Emolientes/lubrificantes } & $\begin{array}{l}\text { Bisacodil, } \\
\text { Picossulfato de sódio } \\
\text { Sene }\end{array}$ \\
\hline & $\begin{array}{l}\text { Docusato de sódio } \\
\text { Óleo mineral } \\
\text { Parafina líquida }\end{array}$ \\
\hline
\end{tabular}

Fonte: Adaptado de CORADAZZI; TARDELLI; CAPONERO, 2019.

Além disso, diarreia é frequente em pacientes que se encontram nos cuidados paliativos, quando a qualidade de vida e o controle adequado dos sintomas é prioridade. Por mais que seja um sintoma frequente, a diarreia é menos comum do que a constipação em pacientes com doença avançada. Menos de 10\% dos pacientes com câncer admitidos no hospital ou em unidades de cuidados paliativos apresentam diarreia; sendo esta definida pela passagem de três ou mais fezes não formadas em um período de 24 horas. As causas mais frequentes de diarreia que pacientes em cuidados paliativos apresentam são: desbalanço da terapia laxativa; drogas como os antibióticos e antiácidos; impactação fecal com diarreia por transbordamento; radioterapia abdominal ou pélvica; outras doenças concomitantes; hábitos alimentares prévios; tumores de cólon, reto, pelve e pâncreas, tumor carcinoide e fistulas (ANCP, 2012; CORADAZZI; TARDELLI; CAPONERO, 2019; FUENTES et al., 2017; ZOCCOLI et al., 2019). 
Esse sintoma pode ser classificado em agudo ou crônico, quando é presente por mais de quatro semanas. Por conta disto, a diarreia é muito debilitante, principalmente nos pacientes com doença avançada. Além da perda de fluidos e eletrólitos e do esforço repetido para ir ao banheiro, os pacientes também vivenciam privações em suas vidas diárias, como ansiedade causada pela incontinência fecal, mudanças emocionais e isolamento social (CORADAZZI; TARDELLI; CAPONERO, 2019).

O manejo para o tratamento da diarreia deve primeiro concentrar-se em encontrar causas específicas que possam justificar o sintoma, para que ela possa ser tratada de forma mais adequada. Porém, em muitos casos, não se reconhece o agente causal da diarreia, mas, da mesma forma, é importante reduzir os sintomas com terapias empíricas. Para tanto, são utilizados medicamentos opioides como a loperamida, codeína e a morfina; outros medicamentos como a clonidina, carbonato de cálcio e ondansetrona também são utilizados. O uso do probiótico Lactobacillus spp. também cresceu bastante nos últimos anos para o manejo desse sintoma (BEZERRA DE LIMA; CARNEIRO ALVES PEREIRA, 2017; CORADAZZI; TARDELLI; CAPONERO, 2019).

Os opioides são seguros e considerados necessários para o tratamento sintomático de diarreia. No entanto, a alta dose de loperamida pode causar arritmia e morte. Já a codeína pode causar sintomas centrais, como sedação ou tontura, mas estes efeitos são raros quando se utiliza loperamida, por esses motivos é de extrema importância que as intervenções medicamentosas tenham uma abordagem correta e premente, permitindo ao paciente seguir seu trajeto sem obstáculos adicionais (CORADAZZI; TARDELLI; CAPONERO, 2019).

\section{Manejo do delirium}

O delirium é um problema muito comum em pacientes com câncer avançado, ocorre em 15\% a $30 \%$ dos pacientes hospitalizados, e em até $85 \%$ dos pacientes na fase terminal. Pode causar estresse aos pacientes e familiares, podendo até interferir em outros sintomas que estão sendo tratados. $\mathrm{O}$ delirium e a demência apresentam sinais comuns, por isso, é importante esclarecer a diferença entre eles. Pacientes com delirium tornam-se irritáveis e podem perder a consciência com o tempo, já a demência desenvolve-se gradualmente, e seu impacto na memória e na consciência é permanente (FARRIOLS DANÉS et al., 2016; FUENTES et al., 2017).

O delirium apresenta-se em três tipos: o hipoativo, quando o paciente permanece maior parte do tempo dormindo ou fechado em si mesmo; hiperativo, quando a pessoa fica agitada, retratando delírios ou alucinações; e o misto. Os sintomas mais comuns incluem: depressão, alucinações, agitação, ansiedade, distúrbio do sono, irritabilidade, problemas de memória, pensamento e fala desorganizada, desorientação e inversão do dia pela noite (ZOCCOLI et al., 2019).

O diagnóstico é clínico e não existem testes ou exames complementares específicos. No entanto, testes para verificar habilidades motoras, níveis de memória e concentração podem ser muito 
úteis. É importante identificar a causa do delirium para fazer a melhor definição de escolha do manejo. Medicamentos, como analgésicos e quimioterápicos, retirada da medicação, falta de oxigênio no sangue e outros distúrbios cerebrais podem ser algumas das causas (FUENTES et al., 2017; GOUVEIA; JOMAR; VALENTE, 2019).

Assim, o principal objetivo do tratamento do delirium é manter o paciente confortável e seguro. Algumas das medidas não farmacológicas incluem: promover ambiente tranquilizado para o paciente, quarto silencioso e bem iluminado. As intervenções farmacológicas podem reduzir o estresse, desconforto e comportamento perigoso do paciente. As medicações não tratam o delirium, mas sim reduzem a agitação impactante do paciente. Em cuidados paliativos há poucos medicamentos para o controle desta condição, por isso, devem ser monitorados os possíveis efeitos adversos. São utilizados antipsicóticos típicos, como o haloperidol e a clorpromazina e os mais utilizados dos atípicos são a olanzapina (está relacionada com maior sedação), risperidona (possui hipotensão ortostática como efeito adverso e sintomas extrapiramidais) e a quetiapina (possui hipotensão ortostática e sedação como efeitos adversos). Contudo, deve-se evitar benzodiazepínicos no delirium, pois aumentam a frequência e pioram os sintomas desse distúrbio (CERVEIRA et al., 2017; ZOCCOLI et al., 2019).

\section{Manejo do distúrbio hemorrágico}

O distúrbio hemorrágico ocorre em cerca de $10 \%$ dos pacientes com câncer avançado, e a maioria dos pacientes evolui a óbito. As causas do sangramento em pacientes com doença avançada variam e podem ter diversas causas e/ou fatores agravantes. Entre as causas estão a invasão tumoral local nos vasos sanguíneos; sangramento superficial do tumor; mucosite; disfunção plaquetária; insuficiência hepática. Ademais, medicamentos como anticoagulantes, antiagregantes plaquetários, trombolíticos, corticoides, ou ainda doenças associadas como a cirrose podem ser fatores relevantes. A equipe de cuidados paliativos deve estar sempre vigilante quanto a sangramentos, e deve sempre interromper o uso dos fármacos desencadeadoras para preveni-los (ZOCCOLI et al., 2019).

Os distúrbios hemorrágicos podem ser visíveis ou internos, contínuos ou intermitentes, de foco localizado ou de focos múltiplos (como nas coagulopatias). Por isso, é necessário um plano de cuidados adequado aos pacientes com risco de hemorragia, atentando-se aos fatores de risco. O planejamento deste cuidado deve ser sempre individualizado, levando em consideração as comorbidades de cada paciente. Para o manejo adequado, além das causas e da apresentação clínica, outros fatores como disponibilidade de recursos, gravidade da doença, expectativa e qualidade de vida, terapias previas, preferencias do paciente e da família e risco-benefício do tratamento devem ser considerados (SANTOS et al., 2017).

O tratamento depende da intensidade do sangramento e do prognóstico prévio do paciente. As medidas não farmacológicas incluem: usar lençóis de cor escura a fim de reduzir angústias associadas à presença do sangue, para conter o sangue são utilizadas compressas ou curativos no local. 
Para as medidas farmacológicas, os medicamentos mais utilizados são o ácido aminocapróico, ácido tranexâmico, etamsilato e, ainda, a vitamina K. A transfusão de sangue pode ser indicada desde que o paciente apresente sintomas relacionados a anemia aguda, e desde que o seu prognóstico definido previamente assim o permitir (COVARRUBIAS-GÓMEZ et al., 2018).

\section{Manejo da dor}

A International Association for the Study of Pain define a dor como uma experiência sensitiva e emocional desagradável, ou semelhante àquela associada, a uma lesão tecidual real ou potencial. É um sintoma comum em oncologia e tem prevalência de até $90 \%$ em pacientes com a doença em estágio avançado. O sucesso para o melhor tratamento da dor requer uma boa avalição inicial, sendo muito importante identificar e compreender os seus diferentes tipos e intensidades. A dor vai além do físico e impõe limitações no estilo de vida do paciente. Fatores psicossociais, espirituais e familiares podem contribuir para redução ou persistência desse sintoma (OLIVEIRA; MEDEIROS, 2020; SRINIVASA et al., 2020; TREEDE, 2018).

A dor é o principal fator responsável pela diminuição da qualidade de vida dos pacientes com câncer. Há diferentes maneiras de classificar a dor, para cada tipo ou intensidade deve-se realizar uma terapia diferenciada. Desta forma, o manejo da dor em pacientes paliativos exige uma abordagem multidisciplinar que contemple o conhecimento da sua fisiopatologia. Para o alívio e a promoção de conforto do paciente existem intervenções essenciais a serem seguidas, como, por exemplo, o plano proposto pela Organização Mundial da Saúde (OMS), que preconiza o uso de medicamentos de acordo com uma escada de analgésicos. A escada é formada por quatro degraus, que contém dor leve, dor moderada, dor intensa e ainda dor refratária (Figura 1). O quarto degrau da escada é o intervencionista, no quão são abarcados os procedimentos neurocirúrgicos, mas eles são indicados nos casos em que os tratamentos nos degraus inferiores não proporcionaram melhora satisfatória ou causaram efeitos adversos não controláveis, e inclui o uso de bloqueios de nervos, raquianestesia (epidural e subaracnoide), administração de anestésicos locais, opioides, agonistas alfa-2, estimulação da medula espinhal e intervenções cirúrgicas, conforme ditado pela condição do paciente (GUERRA et al., 2019; MIGUEL, 2000; NAIME, 2013; OLIVEIRA; MEDEIROS, 2020).

Além do uso da escada analgésica, a avaliação da dor também é essencial. Localização, radiação, intensidade, mudanças no tempo e fatores relacionados à melhoria ou piora dos sintomas devem ser consideradas. É importante investigar se as síndromes dolorosas estão relacionadas ao câncer per se ou ao tratamento. Dessa forma, a equipe poderá tratar cada paciente com dor de forma adequada e individualizada. Para esta avaliação pode-se usar a escala visual analógica (EVA), que tem como propósito descrever a dor com base no relato do paciente, a qual se resulta em uma pontuação que pode variar de 0 a 10, onde 0 a 2 é a dor considerada leve, de 3 a 7 é considerada dor moderada, e de 
8 a 10 é considerada dor intensa. Além do mais, a dor também pode ser avaliada comparando a expressão facial do paciente, conferindo com a escala da face (se está sorridente, triste, fazendo careta, com expressão de dor). Estas são ferramentas de fácil utilização e grande relevância para nortear a equipe quanto às escolhas terapêuticas, permitindo classificar os tipos e intensidades da dor, e a partir desta análise, são escolhidos os métodos terapêuticos (GUERRA et al., 2019; NASCIMENTO, 2017; OLIVEIRA; MEDEIROS, 2020).

Figura 1 - Escada analgésica para o manejo da dor.

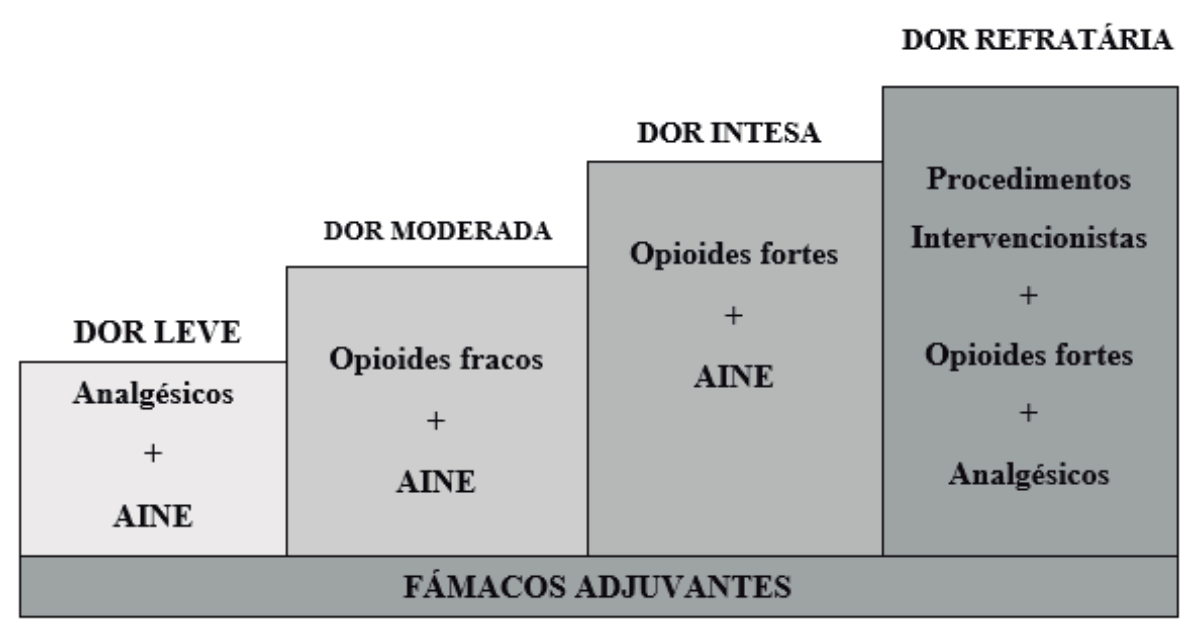

Fonte: Adaptado de OLIVEIRA; MEDEIROS, 2020.

Em relação ao manejo farmacoterapêutico deste sintoma, inicia-se, então, com analgésicos simples, escalonados, e posteriormente para opioides fracos e, por fim, utiliza-se opioides fortes, como a morfina e a metadona. De acordo com o grau da dor, a escada proposta pela OMS deve ser utilizada, quando a dor é de leve a moderada, devem ser utilizados analgésicos não opiáceos e/ou AINES (anti-inflamatório não esteroidal) associados à medicação adjuvante. Em casos de dor moderada, a orientação é prescrever analgésico e/ou AINES, com opiáceo fraco mais adjuvante. Já em casos de dor intensa deve-se utilizar opiáceos fortes mais adjuvantes (GUERRA et al., 2019; OLIVEIRA; MEDEIROS, 2020; PEREIRA et al., 2015).

Em um estudo feito por Guerra et al. (2019), observou-se que para pacientes com dor leve, as instituições costumam fazer o manejo farmacoterapêutico com dipirona e paracetamol. Entre os pacientes considerados moderadamente dolorosos, os principais medicamentos de escolha são o tramadol e o paracetamol, que podem ser associados à codeína. Na dor intensa, a morfina ou metadona podem ser utilizadas sozinhas ou em combinação com algum analgésico. Além destes fármacos em todas as intensidades da dor são utilizados também antidepressivos, anticonvulsivantes, corticosteroides e neurolépticos como medicamentos adjuvantes para o controle do sintoma. A indicação primária desses fármacos não é analgesia, mas quando usados em associação têm efeito coanalgésico em todos os degraus da escada recomendada pela OMS (Quadro 2). Além da terapia com fármacos no manejo 
desse sintoma, também são utilizadas medidas não farmacológicas, como técnicas de relaxamento; terapia física por aplicação de calor ou frio; acupuntura; massoterapia e neuroestimulação. Todas essas técnicas visam propor o melhor controle do sintoma, otimizando a qualidade de vida dos pacientes (BAZANTE, 2016; DE SOUSA-MUÑOZ et al., 2015; GUERRA et al., 2019; PEREIRA et al., 2015).

Quadro 2 - Medicamentos mais comumente utilizados na escala analgésica.

\begin{tabular}{|l|l|}
\hline \multicolumn{1}{|c|}{ CLASSE } & \multicolumn{1}{|c|}{ FÁRMACO } \\
\hline \multirow{3}{*}{ Aines } & $\begin{array}{l}\text { Ácido Acetil Salicílico } \\
\text { Dipirona } \\
\text { Paracetamol } \\
\text { Diclofenaco }\end{array}$ \\
\hline \multirow{2}{*}{ Opioides fracos } & $\begin{array}{l}\text { Codeína } \\
\text { Tramadol }\end{array}$ \\
\hline \multirow{2}{*}{ Opioides fortes } & $\begin{array}{l}\text { Morfina } \\
\text { Metadona } \\
\text { Fentanil }\end{array}$ \\
\hline & $\begin{array}{l}\text { Amitriptilina (antidepressivo) } \\
\text { Carbamazepina (anticonvulsivante) } \\
\text { Dexametasona (corticoide) } \\
\text { Haloperidol (neuroléptico) } \\
\text { Gabapentina (anticonvulsivante) }\end{array}$ \\
\hline
\end{tabular}

Fonte: Adaptado de BAZANTE, 2019; GUERRA et al., 2019.

O uso prolongado de medicamentos pode acentuar efeitos adversos e/ou aumentar a suscetibilidade para as interações medicamentosas. Alguns fatores como idade avançada, uso de muitos medicamentos, função renal/hepática comprometida, mais de um prescritor e comorbidades podem predispor o paciente a interações. Nesse contexto, o uso de opioides, fármaco mais utilizado para o controle do sintoma da dor, embora seja incomum, está associado a quadros clínicos como transtornos do uso de opioides, manifestados pela dificuldade de controle de uso, tolerância e dependência física. Além disso, o uso destes fármacos pode cursar com alterações depressão respiratórias, constipação intestinal, náuseas e vômitos, sedação e delirium. Em pacientes que estão em cuidado paliativo devido à progressão da doença ou à tolerância à medicação, doses maiores poderão ser necessárias, dessa maneira a equipe deve ser cautelosa e estar alerta para ocorrências desses efeitos adversos e/ou interações medicamentosas (OLIVEIRA; MEDEIROS, 2020; ZOCCOLI et al., 2019).

\section{VIAS DE ADMINISTRAÇÃO DE FÁRMACOS EM PACIENTES PALIATIVOS}

O cuidado paliativo busca a qualidade de vida e a dignidade aos pacientes cuja doença não responde mais ao tratamento curativo, dando-se, dessa forma, primazia ao cuidar, propiciando mais conforto ao paciente. A via de administração de fármacos de eleição em cuidados paliativos é a via oral, por ser uma via simples e não invasiva, porém pacientes oncológicos, em situação de 
incurabilidade, demandam de uma via alternativa para a administração de fármacos (NOVELLI et al., 2019; SILVA; HOLANDA, 2019).

Uma das vias que tem sido considerada importante terapêutica, principalmente em pacientes idosos paliativos é a hipodermóclise ou terapia subcutânea, pois oferece conveniência e segurança. A hipodermóclise é uma técnica utilizada na preparação de fluídos e medicamentos por meio do tecido subcutâneo do paciente para fins terapêuticos. Entre as principais vantagens deste método destacam-se o baixo custo do procedimento, a maior facilidade para manejo, maior segurança contra infecções locais e eventos adversos (PONTALTI et al., 2017).

\section{CONSIDERAÇÕES FINAIS}

A partir dos dados de literatura selecionados, é possível afirmar o quão importante é oferecer o cuidado paliativo para o tratamento de sinais e sintomas no câncer terminal. Essa assistência preconiza humanizar a relação da equipe de saúde com o paciente e a família e o tratamento busca proporcionar uma resposta adequada para os pacientes que sofrem com doenças que ameaçam a continuidade da vida, desde o diagnóstico até os momentos finais. Ao longo do desenvolvimento deste artigo, foram evidenciados a importância do farmacêutico na equipe de cuidados paliativos, a fim de contribuir e otimizar no plano de cuidados dos pacientes. Além disso, verificou-se os benefícios dos tratamentos farmacológicos para o manejo dos seguintes sinais e sintomas: manejo dos problemas respiratórios; manejo da náusea e vômito; manejo da constipação e da diarreia; manejo do delirium; manejo dos distúrbios hemorrágicos e o manejo da dor de pacientes oncológicos em cuidado paliativo.

Continuamente, o câncer e o seu tratamento resultam em um certo grau de impacto na qualidade de vida do paciente. Por isso, os cuidados paliativos levam em consideração não só questões físicas e psicológicas, mas também sociais e até espirituais. É importante, ainda, analisar e avaliar os sinais e os sintomas de cada paciente para melhor prescrever e gerenciar cada medicamento, visando uma melhor compreensão das alternativas terapêuticas para que as decisões em saúde sejam tomadas de forma a obter a melhor eficiência no controle do câncer associada ao menor prejuízo possível para o paciente.

Nesse sentido, faz-se cada vez mais necessário desenvolver nas instituições de ensino de graduação e pós-graduação, disciplinas e conteúdos programáticos sobre as principais medicações utilizadas no tratamento dos sinais e sintomas dos pacientes oncológicos que estão em cuidados paliativos. Isso colaborará para uma melhor assistência aos pacientes sob cuidados em terminalidade de vida, além de melhor qualificar os profissionais, melhorar o ambiente onde a assistência é prestada e assegurar o sucesso do tratamento, contribuindo assim de uma forma positiva na melhora da qualidade de vida de cada paciente. 


\section{REFERÊNCIAS}

ALMEIDA, R. G. L. et al. O Manejo da Êmese em uma Unidade Oncológica: a Necessidade da Intervenção Farmacêutica em Tempo Real. Revista Brasileira de Cancerologia, v. 61, n. 2, p. 115-121, 2015.

ALVES, A. R. M. V. Dispneia em cuidados paliativos num serviço de medicina interna: o que informam os registos de enfermagem. Lisboa, 2016. Disponível em: https://bit.ly/392Y43K

ALVES, A. R. M. V.; PINA, P. S. DOS R. S. Dispneia em cuidados paliativos: registos de enfermagem e a autoavaliação da dispneia. Revista de Enfermagem Referência, v. 17, n. 16, p. 53-62, 2018.

ANCP, A. N. DE C. P. Manual de cuidados paliativos ANCP. 2. ed. São Paulo: ANCP, 2012.

ANDRADE, F. M. Emese induzida pela quimioterapia - ainda um problema? [s.1.] Universidade do Porto, 2018.

BAZANTE, P. H. S. Terapia da dor para pacientes oncológicos. Recife: Instituto Nacional de Ensino Superior e Pesquisa e ao Centro de Capacitação Educacional, 2016.

BEZERRA DE LIMA, M.; CARNEIRO ALVES PEREIRA, M. Constipação intestinal em pacientes tratados com opioides: uma revisão integrativa. Revista Brasileira em promoção da Saúde, v. 30, n. 2 , p. $275-282,2017$.

CALADO, D. DOS S.; TAVARES, D. DE H. C.; BEZERRA, G. C. O papel da atenção farmacêutica na redução das reações adversas associados ao tratamento de pacientes oncológicos. Revista Brasileira de Educação e Saúde, v. 9, n. 3, p. 94-99, 2019.

CERVEIRA, C. C. T. et al. Delirium in the elderly: A systematic review of pharmacological and non-pharmacological treatments. Dementia e Neuropsychologia, v. 11, n. 3, p. 270-275, 2017.

CORADAZZI, A. L.; TARDELLI, M.; CAPONERO, R. Cuidados paliativos: diretrizes para melhores práticas. 1. ed. São Paulo - SP: Summus Editorial, 2019.

COVARRUBIAS-GÓMEZ, A. et al. Guía Latinoamericana de Práctica Clínica para el abordaje de la hemorragia masiva en el enfermo paliativo en situación de terminalidade. Revista Mexicana de Anestesiología, v. 41, n. 4, p. 301-311, 2018. 
CRUZ, Â.; OLIVEIRA, C.; CAPELAS, M. L. Instrumentos de avaliação da dispneia e tosse em Cuidados Paliativos: Revisão Sistemática da Literatura. Cuidados Paliativos, v. 4, n. 1, p. 50-64, 2017.

DE SOUSA-MUÑOZ, R. L. et al. Prevalência de dor e adequação da terapêutica analgésica em pacientes internados em um hospital universitário. Medicina (Brazil), v. 48, n. 6, p. 539-548, 2015.

FARRIOLS DANÉS, C. et al. Prevalencia del delirium en pacientes con enfermedad oncológica avanzada ingresados en una unidad de cuidados paliativos. Medicina Paliativa, v. 23, n. 4, p. 165-171, 1 out. 2016.

FRANKEN, L. G. et al. Pharmacokinetic considerations and recommendations in palliative care, with focus on morphine, midazolam and haloperidol. Expert Opinion on Drug Metabolism and Toxicology, v. 12, n. 6, p. 669-680, 2016.

FUENTES, C. et al. Delirium en el paciente hospitalizado. Revista Médica Clínica Las Condes, v. 28 , n. 6 , p. 855-865, 2017.

GOMES, A. L. Z.; OTHERO, M. B. Cuidados paliativos. Estudos Avançados, v. 30, n. 88, p. 155-166, 2016.

GOUVEIA, B. R.; JOMAR, R. T.; VALENTE, T. C. Delirium in cancer patients admitted to the intensive care unit: A retrospective study. Revista Brasileira de Terapia Intensiva, v. 31, n. 4, p. 536-540, 2019.

GUERRA, É. D. et al. Principais medicações utilizadas em cuidados paliativos - Revisão de literatura. Brazilian Journal of Development, v. 5, n. 11, p. 26862-262876, 2019.

KOHLER, L. B.; CERCHIARO, A. C. B.; LEVITES, M. R. Cuidados paliativos ambulatoriais e qualidade de vida em pacientes oncológicos. Diagn. tratamento, v. 21, n. 3, p. 101-105, 2016.

KREMER, D. Prevenção de interações medicamentosas no uso de antineoplásicos: instrumentos para a segurança do pacienteFlorianópolis, 2015. Disponível em: https://bit.ly/392YjvG

LANZA, C. C. DE LA et al. Programa de medicina y cuidados paliativos. Gaceta Mexicana de Oncología, v. 14, n. 3, p. 171-175, 2015. 
LEWIS, S. J.; HEATON, K. W. Stool form scale as a useful guide to intestinal transit time. Scandinavian Journal of Gastroenterology, v. 32, n. 9, p. 920-924, 1997.

LOBATO, L. C. et al. Cuidados farmacêuticos no tratamento oncológico: uma revisão de literatura. Conexão ciência, v. 14, n. 1, p. 31-38, 2019.

MIGUEL, R. Interventional treatment of cancer pain: The fourth step in the world health organization analgesic ladder? Cancer Control, v. 7, n. 2, p. 149-156, 2000.

NAIME, F. Manual de Tratamento da Dor, 2013. Disponível em: https://bit.ly/31rnKwP

NASCIMENTO, J. C. C. Avaliação da dor em paciente com câncer em cuidados paliativos a luz da literatura. Saúde \& Ciência em ação, v. 3, n. 01, p. 11-26, 2017.

NOGUEIRA, P. Abordagem terapêutica do doente paliativo com insuficiência hepática e/ou renal. [s.1.] Universidade de Coimbra, 2019.

NOVELLI, B. T. et al. Recomendações para utilização da hipodermóclise em pacientes sobre cuidados paliativos. Revista Enfermagem em Evidência. v. 3, n. 1, p. 139-153, 2019.

OLIVEIRA, E. P.; MEDEIROS, P. Cuidados paliativos em pneumologia. Jornal Brasileiro de pneumologia. v. 46, n. 3, p. 1-11, 2020.

PEREIRA, D. T. DE S. et al. Condutas terapêuticas utilizadas no manejo da dor em oncologia. Revista de Pesquisa: Cuidado é Fundamental Online, v. 7, n. 1, p. 1883-1890, 2015.

PONTALTI, G. et al. Benefícios da Hipodermóclise na Clínica Paliativa de Pacientes com Câncer: Relato de Caso. Revista Brasileira de Cancerologia, v. 62, n. 3, p. 247-252, 2017.

RAGGHIANTI, K. C. Avaliação do uso de antieméticos em quimioterapia. Monografia apresentada ao Programa de Aprimoramento Profissional/CRH/SES-SP, Ribeirão Preto, 2017.

RAMOS, A. F. N.; TAVARES, A. P. M.; MENDONÇA, S. M. S. Pain and dyspnea control in cancer patients of an urgency setting: nursing intervention results. Revista Dor, v. 18, n. 2, p. 166-172, 2017. 
ROBERTS, D. M. et al. Clinical pharmacokinetics in kidney disease application to rational design of dosing regimens. Clinical Journal of the American Society of Nephrology, v. 13, n. 8, p. 1254-1263, 2018.

SANTOS, M. et al. Diretrizes oncológicas. 1. ed. Rio de Janeiro: Elsevier, 2017. v. 42

SILVA, S. M. DE O.; HOLANDA, M. DE A. Prática de hipodermóclise nos pacientes oncológicos em cuidados paliativos. Gepnews, v. 2, n. 2, p. 383-389, 2019.

SOUZA, M. et al. Atuação do farmacêutico hospitalar na oncologia. Boletim Informativo Geum, v. 7, n. 1, p. 54-63, 2016.

SRINIVASA, N. R. et al. Definição revisada de dor pela Associação Internacional para o Estudo da Dor : conceitos, desafios e compromissos. Revista SBED, p. 1-8, 2020.

TEIXEIRA, M. C. G. Uma atualização do artigo publicado no livro: farmácia clínica e atenção farmacêutica, faculdade de ciencias farmacêuticas, Universidade de São Paulo-Brasil. [s.l.] Universidade de Coimbra, 2015.

TREEDE, R. D. The International Association for the Study of Pain definition of pain: As valid in 2018 as in 1979, but in need of regularly updated footnotes. Pain Reports, v. 3, n. 2, p. 3-5, 2018.

WHO. Palliative Care. Disponível em: https://bit.ly/3EfsD19. Acesso em: 24 nov. 2020.

ZOCCOLI, T. L. V. et al. Desmistificando cuidados paliativos um olhar multidisciplinar. 1. ed. Brasília: Oxigênio, 2019. 